\title{
ON THE BLOCH-LANDAU CONSTANT FOR SCHLICHT FUNCTIONS
}

\author{
RUTH E. GOODMAN
}

The definition of the Bloch-Landau constant $\mathfrak{A}$ is based upon the following theorem due to Bloch:1

THEOREM 1. There is an absolute positive constant $P$ with the following property: Let $f(x)$ be regular for $|x|<1,\left|f^{\prime}(0)\right|=1$. Then the map of $|x|<1$ under $f(x)$ contains in a single sheet an open circle of radius $P$.

In addition to the original Bloch constant $\mathfrak{B}$, the least upper bound of the $P$ satisfying the above theorem, Landau ${ }^{2}$ has defined two other constants, $\mathfrak{R}$ and $\mathfrak{A}$, in connection with this theorem. $\mathfrak{l}$ is the least upper bound of the $P$ of Theorem 1 if the requirement that there

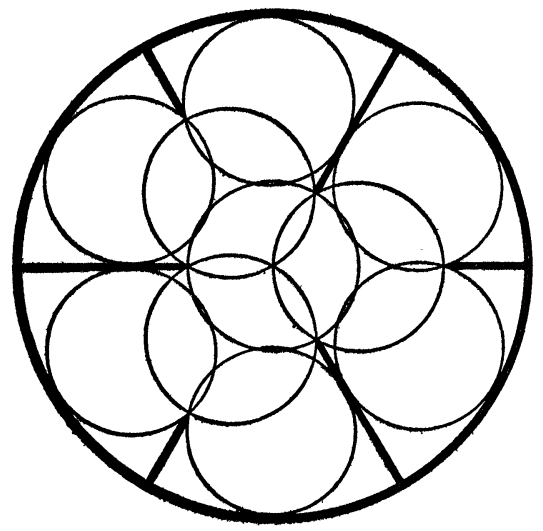

FIG. 1

be a circle of radius $P$ contained in a single sheet is replaced by the requirement that there be a circle of radius $P$ each point of which is an interior point of some sheet of the map. $\mathfrak{A}$ is the least upper bound of the $P$ of Theorem 1 if it is required that the function $f(x)$ be schlicht for $|x|<1$. It is immediately apparent that $\mathfrak{B} \leqq \mathfrak{R} \leqq \mathfrak{A}$.

Received by the editors August 14, 1944.

${ }^{1}$ Bloch, Les theorèmes de $M$. Valiron sur les fonctions entières, et la theorie de l'uniformisation, C. R. Acad. Sci. Paris vol. 178 (1924) pp. 2051-2052.

$2 \mathrm{E}$. Landau, Über die Blochsche Konstante und zwei verwandte Weltkonstanten, Math. Zeit. vol. 30 (1929) pp. 608-634. This paper includes a proof of Theorem 1. 
The exact value of none of these three constants is known, although upper and lower bounds have been established for all three constants. Landau ${ }^{2}$ proved that $\mathfrak{R}<\mathfrak{A}$; that $\mathfrak{B}<\mathfrak{R}$ was proved by Ahlfors. ${ }^{\mathbf{3}}$

The best upper bound given so far for the constant $\mathfrak{A}$ is due to Robinson :4 $\mathfrak{A}<0.658$. This result was obtained by mapping $|x|<1$ on to a circle with six radial slits, three each of two different lengths, symmetrically placed. (See Fig. 1.) The lengths of the slits are determined so as to make the ten circles shown in the region all maximal.

Robinson's procedure is capable of extension to an infinite sequence of mappings, which can be successfully exploited. The $n$th region of this sequence (see Fig. 2) is that portion of the slit plane enclosed by the circle $|y|=R_{n}$. The radial slits of the $n$th region fall along the lines through the origin and the $3 \cdot 2^{n-1}$ st roots of unity. The $R_{n}$ are determined so that the maximal circles in every region shall be of radius unity.

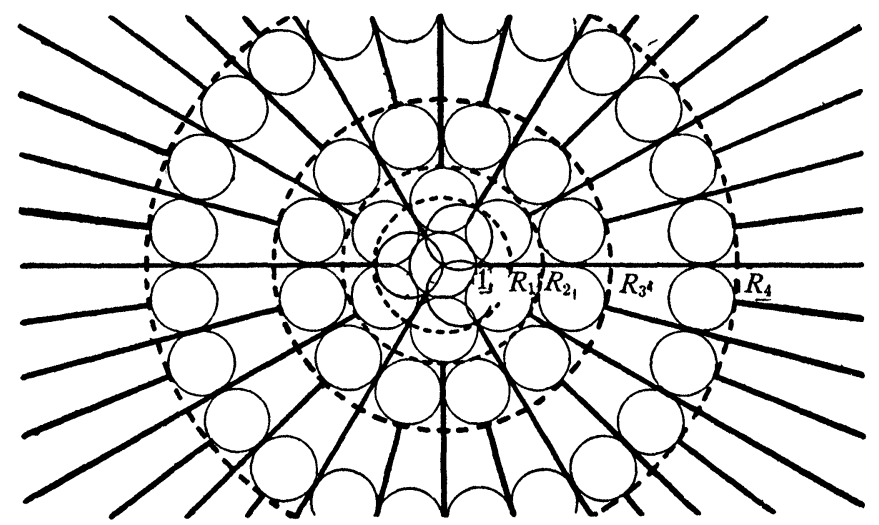

FIG. 2

A similar sequence of regions may be defined for every integer $s \geqq 3$, by beginning with $s$ slits falling along the $s$ lines through the origin and the sth roots of unity, and ending on the circle $|y|=1$. Call the regions of this sequence $C_{1,8}, C_{2,8}, C_{3,8}, \cdots$.

(Note that the region $C_{2,3}$, although it contains two sets of three slits each, is not exactly the region $D$ of Fig. 1 . The region $C_{2,3}$ contains only four maximal circles, since it is bounded by the circle $|y|=R_{2}=2.9557$, which passes through the ends of the third set of

${ }^{3}$ L. V. Ahlfors, An extension of Schwarz's lemma, Trans. Amer. Math. Soc. vol. 43 (1938) pp. 359-364.

4 R. M. Robinson, The Bloch constant $\mathfrak{A}$ for a schlicht function, Bull. Amer. Math. Soc. vol. 41 (1935) pp. 535-540. 
slits. The circle $|y|=2.9561$ which bounds $D$ is tangent to the six outer maximal circles in $D$.)

Let $y=f_{n, 8}(x), f_{n, 8}(0)=0$, be the function which maps $|x|<1$ on to $C_{n, s}(s \geqq 3 ; n=1,2,3, \cdots)$.

It is clear that the upper bound $B_{n, s}$ for $\mathfrak{A}$ furnished by mapping $|x|<1$ on to $C_{n, 8}$ is

$$
\mathfrak{U} \leqq B_{n, s}=1 /\left|f_{n, s}^{\prime}(0)\right| .
$$

Theorem 2. Let $f(x)$ and $g(x)$ be regular for $|x|<1, f(0)=g(0)=0$. Let $f(x)$ map $|x|<1$ on to $F$ and $g(x)$ map $|x|<1$ on to $G$, where $F \subset G$. Then $\left|f^{\prime}(0)\right|<\left|g^{\prime}(0)\right|$.

This result is readily proved by noticing that the function $g^{-1}(f(x))$ satisfies the conditions of Schwarz's lemma. ${ }^{5}$

Since $C_{1,8} \subset C_{2,8} \subset C_{3,8} \subset \cdots$, it is clear, by Theorem 2, that the sequence $\left\{B_{1, s}, B_{2, s}, B_{3, s}, \cdots\right\}$ is monotonically decreasing to a positive limit $B_{\infty, 8}$.

THEOREM 3. If $s>3$, then $B_{\infty, 8}>B_{\infty, 3}$.

PROOF. Let $P_{1,8}$ be the entire plane with the $s$ slits of the region $\sum_{j=1}^{\infty} C_{j, 8}$ which end on the unit circle. Let $y=g(x), g(0)=0$, map $|x|<1$ on to $P_{1,8}$. Now $P_{1,8} \supset C_{n, 8}$ for fixed $s$ and every $n$. By Theorem 2 it follows that

Since the function

$$
B_{\infty, 8} \geqq 1 /\left|g^{\prime}(0)\right|
$$

$$
k(x)=x /(1-x)^{2}
$$

transforms $|x|<1$ into the entire plane slit along the negative real axis from $-\infty$ to $-1 / 4$, therefore

whence

$$
g(x)=\left(-4 k\left(x^{8}\right)\right)^{1 / s},
$$

Thus

$$
\left|g^{\prime}(0)\right|=4^{1 / s} \text {. }
$$

$$
1 /\left|g^{\prime}(0)\right|=1 / 4^{1 / 8}>0.707
$$

By Theorem 2, $B_{\infty, 3}<B_{2,3}$, and the value $B_{2,3}<0.658$ is found by computation. (For the method of computation, see reference 4.) Combination of the above inequalities gives Theorem 3 .

Since the case $s=3$ is the only one hereafter of interest, we shall from now on drop the subscript " 3 " from the notations $f_{n, 3}(x), C_{n, 3}$, $B_{n, 3}$. Thus the mapping regions $C_{n}$ are now exactly those of Fig. 2 .

THEOREM 4. $\mathfrak{A} \leqq B_{\infty}<0.65647$.

\footnotetext{
' See L. R. Ford, Automorphic functions, p. 166.
} 
Proof. The function of the sequence $\left\{f_{n}(x)\right\}$ which maps $|x|<1$ on to $C_{4}$ is

$$
f_{4}(x)=R_{4} K^{1 / 12}\left(p_{4} k\left(K^{2}\left(p_{3} k\left(K^{2}\left(p_{2} k\left(-K\left(p_{1} k\left(x^{3}\right)\right)\right)\right)\right)\right)\right),\right.
$$

where

$$
k(x)=x /(1-x)^{2}, \quad K(x)=\text { the inverse of } k(x) .
$$

The constants $p_{j_{-}}$appearing in $f_{4}(x)$ are determined, in terms of the radii $R_{j}$, so as to adjust properly the lengths of the slits in $C_{4}$ :

$$
\begin{aligned}
& p_{4}=-4 k\left(-\left(R_{3} / R_{4}\right)^{12}\right), \\
& p_{3}=4 M\left(\frac{1}{p_{4}} k\left(\left(R_{2} / R_{4}\right)^{12}\right)\right), \\
& p_{2}=4 M\left(\frac{1}{p_{3}} N\left(\frac{1}{p_{4}} k\left(\left(R_{1} / R_{4}\right)^{12}\right)\right)\right), \\
& p_{1}=E /\left(E+p_{2}\right), \quad E=4 N\left(\frac{1}{p_{3}} N\left(\frac{1}{p_{4}} k\left(\left(1 / R_{4}\right)^{12}\right)\right)\right),
\end{aligned}
$$

where

$$
M(b)=\left(4 b^{2}+b\right)^{1 / 2}-2 b, \quad N(b)=\left(4 b^{2}+b\right)^{1 / 2}+2 b,
$$

and where

$R_{0}=1, \quad R_{1}=2, \quad R_{2}=2.9557, \quad R_{n}=1+\csc \left(\pi / 3 \cdot 2^{n-1}\right)$ for $n \geqq 3$.

Since

$$
1 / B_{4}=\left|f_{4}^{\prime}(0)\right|=R_{4} p_{4}^{1 / 12} p_{3}^{1 / 6}\left(p_{2} p_{1}\right)^{1 / 3}
$$

we find

$$
\mathfrak{A} \leqq B_{\infty}<B_{4}<0.65647 \text {. }
$$

It is possible to show that the upper bound of $B_{\infty}$ given in Theorem 4 is accurate to four significant figures, by considering the functions $g_{n}(x), g_{n}(0)=0$, which map $|x|<1$ on to the regions $P_{n}$, defined as follows: Let $P_{n}$ be the entire plane with all those slits of the region $C_{\infty}=\sum_{j=1}^{\infty} C_{j}$ which end on the circles $|y|=R_{k}(k=0,1,2, \cdots, n-1$; $\left.R_{0}=1\right)$. Let $D_{n}=1 /\left|g_{n}^{\prime}(0)\right|$. Then by Theorem 2 the sequence $\left\{D_{n}\right\}$ increases monotonically to a limit $D_{\infty} \leqq B_{\infty}$.

By observing ${ }^{6}$ that the sequences $\left\{f_{n}(x)\right\}$ and $\left\{g_{n}(x)\right\}$ are both normal families on the unit circle, we can conclude that they converge regularly on $|x|<1$ to a common limit function $\phi(x)$. Indeed, $\phi(x)$ maps $|x|<1$ on to $C_{\infty}$, the common kernel of the sequences of regions $\left\{C_{n}\right\}$ and $\left\{P_{n}\right\}$. Thus

${ }^{6}$ See Carathéodory, Conformal representation, chap. 5. 


$$
D_{\infty}=1 /\left|\phi^{\prime}(0)\right|=B_{\infty} \text {. }
$$

It is interesting, however, to note that we can prove this last statement directly, without resort to the more powerful theorems on the behaviour of normal families:

TheOREM 5. $D_{\infty}=B_{\infty}$.

Proof. If $Q_{n}$ is the region into which $C_{n}$ is transformed by the function

$$
u_{n}(x)=4^{1 / \mu} R_{n}\left(-k\left(-\left(x / R_{n}\right)^{\mu}\right)\right)^{1 / \mu} \quad\left(\mu=3 \cdot 2^{n-1}\right),
$$

then $Q_{n} \supset P_{n}$. For $Q_{n}$ is easily seen to be the whole plane with $\mu$ slits along the same lines as the $\mu$ slits of $P_{n}$. Let $y_{0}$ be the end point of one of the slits in $Q_{n}$. The end point of the slit in $P_{n}$ along this same line is some point $R_{h} e^{2 m \pi i / \mu}(0 \leqq h<n ; 0 \leqq m<\mu)$. Now

$$
\left|y_{0}\right|=\left|u_{n}\left(R_{h} e^{2 m \pi i / \mu}\right)\right|=4^{1 / \mu} R_{h} R_{n}^{2} /\left(R_{n}^{\mu}+R_{h}^{\mu}\right)^{2 / \mu}>R_{h},
$$

whence indeed $Q_{n} \supset P_{n}$.

Now the function $w_{n}(x)=u_{n}\left(f_{n}(x)\right)$ transforms $|x|<1$ into $Q_{n}$. Furthermore,

$$
\left|w_{n}^{\prime}(0)\right|=\left|u_{n}^{\prime}(0)\right| \cdot\left|f_{n}^{\prime}(0)\right| \text {. }
$$

Now $\left|u_{n}^{\prime}(0)\right|=4^{1 / \mu}$. Thus, by applying Theorem 2 to the relation $Q_{n} \supset P_{n} \supset C_{n}$, we have

$$
\left|w_{n}^{\prime}(0)\right|=4^{1 / \mu}\left|f_{n}^{\prime}(0)\right|>\left|g_{n}^{\prime}(0)\right|>\left|f_{n}^{\prime}(0)\right| \quad\left(\mu=3 \cdot 2^{n-1}\right) .
$$

But $4^{1 / \mu} \rightarrow 1$ as $n \rightarrow \infty$, whence Theorem 5 is true.

THEOREM 6. $\quad 0.65646<B_{\infty}<0.65647$.

Proof. The function of the sequence $\left\{g_{n}(x)\right\}$ which maps $|x|<1$ on to $P_{3}$ is

$$
g_{3}(x)=\left(q_{3} k\left(K^{2}\left(q_{2} k\left(-K\left(-q_{1} k\left(x^{3}\right)\right)\right)\right)\right)\right)^{1 / 6},
$$

where

$$
q_{3}=4 R_{2}^{6}, \quad q_{2}=4 M\left(R_{1}^{6} / q_{3}\right), \quad q_{1}=4 N\left(1 / q_{3}\right) /\left(4 N\left(1 / q_{3}\right)+q_{2}\right),
$$

where $M(b), N(b), R_{1}, R_{2}$ are as before. Since

$$
1 / D_{3}=\left|g_{3}^{\prime}(0)\right|=q_{3}^{1 / 6}\left(q_{2} q_{1}\right)^{1 / 3},
$$

we have the result stated in the theorem:

$$
0.65646<D_{3}<D_{\infty}=B_{\infty} \text {. }
$$


Robinson has proved ${ }^{7}$ that if $f(x), f^{\prime}(0)=1, f(0)=0$, maps $|x|<1$ on to $R$, where the largest open circle in $R$ has exactly the radius $\mathfrak{A}$, then $R$ has the following property: If the analytic arc $D$ is part of the boundary of $R$, then there are points of $R$ on each side of $D$ in the neighborhood of any point of $D$. It is interesting to note that our best upper bound $B_{\infty}$ for $\mathfrak{A}$ is furnished by a region with this property.

Although the numerical improvement over Robinson's result is not very marked, the mapping of the unit circle $|x|<1$ on the open slit plane $C_{\infty}$ has some features of interest. Each ray through the origin which does not fall on a slit (that is, a ray whose angle with the positive real axis is different from $2 \pi m / 3 \cdot 2^{n}$ ) determines a "prime end." 8 Such a ray is mapped on to a curve which has a well defined end point on the unit circle. Through the introduction of prime ends the mapping of the interior of domains can be extended to the boundary points. 9 The points on the boundary of the unit circle are mapped either into the slits in $C_{\infty}$ or into prime ends. The set $S$ of all points on the unit circle which go over into prime ends is a nowhere dense perfect Cantor set, while the points which are mapped on the slits form denumerably many intervals which lie everywhere dense.

Now the set $S$ is of measure 0 . Indeed, let $y=\phi(x)$ map $|x|<1$ on $C_{\infty}$. Then $\phi(x) \rightarrow \infty$ when $x$ approaches a point of $S$. Thus the function

$$
\Phi(x)=x / \phi(x)
$$

is regular and bounded in $|x|<1$, since on $|x|<1,|\Phi(x)| \leqq 1 / a$, where $a$ is the shortest distance from the origin to a point on a slit. Now by a theorem of Fatou, a regular and bounded function approaches a limit almost everywhere on the unit circle. We can therefore extend $\Phi(x)$ to the boundary (as we have already done indirectly for $\phi(x)$ by the introduction of prime ends). The extended $\Phi(x)$ is 0 on $S$. By a theorem of F. and M. Riesz, ${ }^{10}$ we know that a bounded analytic function, which is not identically 0 , can attain the value 0 on the unit circle in at most a set of measure 0 . Thus $m S=0$.

\section{University of Pennsylvania}

${ }^{7}$ R. M. Robinson, Bloch functions, Duke Math. J. vol. 2 (1936) pp. 458-459.

${ }^{8}$ See C. Carathéodory, UUber die Begrenzung einfach zusammenhangender Gebiete, Math. Ann. vol. 73 (1912) pp. 323-370.

9 See Hurwitz-Courant, Funktionentheorie, pp. 400-405.

${ }^{10} \mathrm{~F}$. and M. Riesz, Über die Randwerte einer analytischen Funktion, Compte Rendu du Quatrième Congrès des mathématiciens scandinaves, Stockholm, 1916, pp. 27-44. See also G. Szegö, Über die Randwerte einer analytischen Funktion, Math. Ann. vol. 84 (1921) pp. 232-244. 